\title{
Las competencias de los graduados y su evaluación desde la perspectiva de los empleadores*
}

\author{
Graduates' competences and assessment from their employers' perspective
}

\author{
Nati Cabrera Lanzo, ${ }^{a}$ María López López, ${ }^{b}$ María Portillo Vidiella ${ }^{c}$ \\ ${ }^{a}$ Edulab del elearn Center de la Universitat Oberta de Catalunya \\ Departamento de Didáctica y Organización Educativa, Facultad de Educación. Universidad de Barcelona \\ Telf.: (34) 93326 3600. Correo electrónico: ncabrera@uoc.edu \\ bDepartamento de Didáctica y Organización Escolar. Facultad Ciencias de la Educación. \\ Universidad de Granada \\ Telf.: (34) 95824 9677. Correo electrónico: mclopez@ugr.es

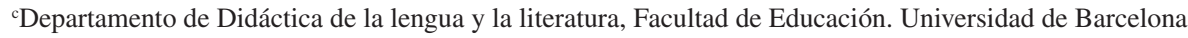 \\ Telf.: (34) 9349607 20. Correo electrónico: mcportillo@ub.edu
}

\begin{abstract}
RESUMEN
El propósito de este artículo es estudiar las percepciones de los empleadores acerca de las competencias que deberían tener los graduados universitarios en el ámbito laboral y de la contribución de la evaluación a su desarrollo. Desde un enfoque metodológico cualitativo y mediante cuestionario abierto, se ha recabado información de empleadores de Educación Primaria, Farmacia e Ingeniería sobre las siguientes cuestiones: competencias que deberían tener los graduados, instrumentos y criterios de evaluación que utilizan en sus procesos de selección y valoración sobre el aprendizaje y la evaluación de competencias en la universidad. Los resultados permiten contrastar percepciones ofrecidas desde los tres sectores investigados. Finalmente, presentamos una reflexión crítica que profundiza en la complejidad del enfoque de competencias y la necesidad de adaptar los sistemas de evaluación. Se aportan sugerencias para estrechar la relación con el mundo laboral, incrementar la empleabilidad y mejorar la calidad de la enseñanza universitaria.
\end{abstract}

Palabras clave: evaluación de competencias, educación superior, graduados universitarios, empleadores.

\section{ABSTRACT}

The purpose of this article is to show the employer's perspective regarding the competences required of college graduates, from different professional sectors and the contribution of evaluation systems to its development and assessment. From a qualitative methodological approach, an open questionnaire has been applied in order to gather information from employers of Primary Education, Pharmacy and Engineering on the following issues: competences that graduates should have; instruments and evaluation criteria used in their selection processes and, learning strategies and assessment systems used at the university. The results allow us contrasting perceptions offered from the three sectors investigated. Finally, we present a critical reflection that explores the complexity of the competencies approach and the need to adapt the evaluation systems to this new scenario. Suggestions to strengthen the relationship with the labor market, increasing employability and improving the quality of university education are provided.

Key words: competence-based assessment, higher education, college graduates, employers.

Este trabajo recoge parte de los resultados del proyecto de investigación que lleva por título "El impacto de la evaluación educativa en el desarrollo de competencias en la universidad. La perspectiva de las primeras promociones de graduados", con referencia EDU2012-32766, financiado por el Ministerio de Economía y Competitividad español. 


\section{INTRODUCCIÓN: JUSTIFICACIÓN Y MARCO TEÓRICO}

Con la reciente creación del Espacio Europeo de Educación Superior (EEES), han sido frecuentes las presiones y demandas que, desde distintos sectores (gobiernos, asociaciones profesionales y empleadores), apelan a la institución universitaria a convertirse en motor de desarrollo económico mediante la revalorización de la formación práctica y la mejora de la empleabilidad de los egresados. Cómo responder a estos requerimientos se ha convertido en un tema clave de la política educativa universitaria y en un interesante foco de indagación en los últimos años (Alonso, Fernández y Nyssen, 2009; McMurray et al., 2011; Parris y Saville, 2011). Sin embargo, en la actualidad, como reconoce Watson (2011), los datos de que disponemos son insuficientes para determinar el verdadero alcance e impacto de los cambios adoptados y de su incidencia en el acceso de los estudiantes al mercado laboral o la mejora de la práctica formativa.

Algunos de los estudios realizados hasta el momento apuntan a resultados satisfactorios y esperanzadores (Nelcy et al., 2012; Taylor y Hooley, 2014), pero un número significativo de ellos insisten en la necesidad de continuar potenciando la relación, aún deficitaria, entre universidad y mundo laboral (Rutt et al., 2013; Torres-Coronas y Vidal-Blasco, 2015).

Trabajos como los realizados por Jackson (2012), Velde (2009) o Kellermann (2007), en el contexto internacional, reflejan la distancia existente entre las competencias adquiridas durante la formación universitaria y las requeridas por el mercado laboral. Jackson (2012) reconoce, apoyándose en los datos extraídos de su investigación, que la percepción que tiene el alumnado respecto a su desarrollo competencial está muy por encima de la que reconocen los empleadores. En opinión de Velde (2009) existe una brecha significativa entre las demandas de las empresas y la formación ofertada en el sistema educativo, lo que motiva que el nivel de expectativas de los empleadores, respecto a las competencias del alumnado egresado, sea bajo.

También Alonso et al. (2009) y Freire, Teijeiro y Pais (2011), en las investigaciones llevadas a cabo en el contexto español, han subrayado las diferencias existentes entre las competencias cultivadas por la universidad y las demandadas por el sector empresarial. Estas discrepancias llevan a estos últimos autores a reconocer abiertamente que "sería necesario que el sistema universitario español mejorase la formación en competencias de los graduados" (Freire et al., 2011, p. 17).

Mejorar la formación práctica de los estudiantes y su desarrollo competencial pasa, en opinión de Parris y Saville (2011), por adoptar tres tipos de medidas: llevar a cabo prácticas en empresas, estrechar vínculos con el mundo laboral y aumentar la presencia de los aspectos vinculados a la práctica profesional en los planes de estudios. Para Villarroel y Bruna (2014), en cambio:

[...] la incorporación de las competencias a la educación superior requiere que los docentes se actualicen en metodologías de enseñanza y evaluación, incorporen actividades prácticas donde observen el despliegue de las competencias esperadas en la asignatura, cuenten con mayor tiempo de preparación de clases, construyan evaluaciones auténticas y entreguen retroalimentación permanente a los estudiantes. (2014, p. 23).

En un reciente estudio llevado a cabo por Pozo y Bretones (2015), en el que responsables de calidad de las titulaciones de distintas universidades españolas han analizado las dificultades y retos que ha supuesto la implantación de los títulos de grado, se ha subrayado 
la necesidad de un replanteamiento de los procesos iniciados vinculados a estos nuevos títulos y de la puesta en marcha de iniciativas que mejoren su evolución.

Resultados y aportaciones como estas invitan a mantener vivo el debate y el compromiso con las líneas de investigación abiertas con la incorporación de las competencias a la educación universitaria. En estos momentos en que se asiste a la acreditación de las primeras promociones de graduados en las titulaciones adaptadas al EEES en España, y que se comienza a disponer de los primeros datos sobre el impacto real de las acciones emprendidas, resulta de vital importancia impulsar procesos de análisis y reflexión que permitan compartir resultados, superar deficiencias y establecer nuevos compromisos desde los que impulsar la mejora.

No cabe duda que los esfuerzos desplegados por las instituciones y docentes universitarios para responder a los desafíos del EEES han sido considerables, tanto en las labores de planificación de las titulaciones y materias, como en su deseo de trasladar las nuevas premisas a la práctica formativa, pero la rigidez de las estructuras administrativas y burocráticas que todavía sustentan la educación superior, la falta de formación docente y las condiciones laborales poco propicias en las que los docentes llevan a cabo la enseñanza (ratios elevadas, trato discriminatorio de las labores docentes en los sistema de evaluación de la calidad docente vigentes, falta de cultura colaborativa, inercia del alumnado, débil coordinación del profesorado, entre otras) son obstáculos que dificultan los cambios que el aprendizaje y evaluación de competencias requiere. Algunas de estas limitaciones han sido también señaladas por Tran (2013, p. 631) cuando afirma que "aunque han evidencias del esfuerzo para cambiar, para incluir y desarrollar competencias en el sistema, en el plan de estudios y mejorar el método de enseñanza tradicional, la pasividad del alumnado y las características culturales que lo envuelven todo, obstaculizan el esfuerzo desplegado para desarrollar competencias en el alumnado universitario". Todo parece indicar que la incorporación del enfoque de competencias a la educación superior es, como ha reconocido Jenkins (2000), un camino largo y difícil y, en el caso del contexto español, una asignatura pendiente (García-San Pedro y Gairín, 2011).

La situación actual en que se encuentra la universidad en estos momentos hace aconsejable ampliar el número de estudios y profundizar en aquellos aspectos que se consideren claves para mejorar la calidad de la formación universitaria. En este sentido, analizar las percepciones que tienen los empleadores sobre las competencias adquiridas y clarificar el papel que, en su opinión, juega la evaluación en el desarrollo de dichas competencias lo es. Este trabajo es, pues, una contribución de gran interés porque la percepción que tienen los profesionales del mercado laboral sobre el nivel competencial del alumnado parece discrepar de la percepción que tienen otros agentes del proceso formativo, como el alumnado (Bhanugopan y Fish, 2009; Jackson, 2012; Torres-Coronas y VidalBlasco, 2015) o el profesorado (Palmer, Montaño y Palou, 2009; Wickramasinghe y Perera, 2010), por lo que conviene contemplar sus valoraciones de cara a la toma de decisiones.

Por otra parte, esta investigación permite explorar un terreno poco transitado en nuestro país que es el vínculo existente entre el desarrollo competencial y el proceso evaluador, en la línea ya iniciada por Baartman et al., (2007) y Gulikers, Baartman y Biemans (2010) en otros contextos. Como ha reconocido Cano (2008):

Algunas titulaciones (por ejemplo, todas aquellas que han sido titulaciones piloto) han avanzado en los diseños por competencias y han estimulado cambios metodológicos. Sin embargo, no todas han previsto un sistema de evaluación por competencias. La evaluación queda, de nuevo, 
relegada a un segundo plano, desgajada de los procesos de enseñanza-aprendizaje, aislada. (2008, p. 2).

Por este motivo, en este trabajo se ha querido profundizar en la opinión que tienen los empleadores sobre los procedimientos de evaluación utilizados para valorar las competencias, cuáles consideran más adecuados y constatar si sus apreciaciones coinciden con las formuladas por los empleadores de otros países (Gulikers et al., 2010; Schomburg y Teichler, 2007). Cuestiones como estas, además de ser útiles para adoptar decisiones estratégicas en el diseño y gestión de las titulaciones y materias, son claves para hacer efectivo y real el cambio que se persigue y que no es otro que mejorar la calidad de la formación universitaria y la inserción profesional de los egresados.

\section{METODOLOGÍA}

Desde un enfoque metodológico cualitativo, la exploración realizada se basa en la percepción y la interpretación de la realidad que se pretende investigar por parte de informantes clave, y que son profesionales expertos en la selección de personal en diversos sectores profesionales. Para la recogida de la información se ha aplicado un cuestionario de preguntas abiertas, previamente validado por expertos, que se ha distribuido, a través del correo electrónico, a once empleadores de tres sectores profesionales: educativo (4), farmacéutico (3) y de ingenierías (4). Para la selección de los empleadores se ha tenido en cuenta que sean profesionales en activo y responsables de la selección de personal o vinculados a los procesos de evaluación de los profesionales para su reclutamiento. En el cuestionario se han planteado las preguntas siguientes:

1. ¿Qué competencias valora usted en un graduado de Educación Primaria / Farmacia / Ingeniería Informática?

2. ¿Qué instrumentos, actividades o estrategias utiliza para evaluar las competencias de un candidato?

3. ¿Cómo cree usted que se podría estimular el desarrollo de las competencias que usted valora más (las más relevantes) desde la universidad?

4. ¿Cómo cree que se deberían evaluar los aprendizajes y las competencias de los/as estudiantes?

5. ¿Considera usted oportuno añadir algo más?

Para el análisis de resultados se ha procedido al análisis descriptivo, agrupando los resultados por temas, a partir de las preguntas planteadas en la investigación, y poniendo en relación la percepción e interpretación de los informantes con la literatura revisada (Colás y Buendía, 1998).

\section{RESULTADOS}

Los resultados obtenidos han sido agrupados en dos grandes bloques: las competencias que los empleadores valoran en los graduados y cómo las observan y evalúan en los candidatos, que recoge la información relativa a las preguntas 1 y 2 , y el punto de vista de 
los empleadores sobre la enseñanza de las competencias en la universidad, que recoge la información relativa a las preguntas 3,4 y 5 .

\subsection{PRIMER BLOQUE DE RESULTADOS: LAS COMPETENCIAS QUE LOS EMPLEADORES VALORAN EN LOS GRADUADOS Y CÓMO LAS EVALÚAN EN LOS CANDIDATOS}

\subsubsection{Las competencias más valoradas por los empleadores en los graduados}

En general, los empleadores encuestados atribuyen a la universidad la responsabilidad de la formación conceptual y técnica de los candidatos, que se corresponderían con las competencias específicas que recoge el proyecto Tuning y que se definen como aquellas competencias específicamente relacionadas con el conocimiento concreto de cada área temática (Proyecto Tuning, 2007).

En este sentido, y siempre en relación a los conocimientos o competencias específicas, los empleadores no suelen evaluarlos, pero sí valoran, como requisito mínimo e indispensable, que los graduados las tengan a través de la comprobación de que están en posesión del título correspondiente. A partir de aquí, los empleadores pasan a centrarse, fundamentalmente, en lo que ellos llaman el perfil personal del candidato: "El requisito de que sea un Licenciado en Farmacia (o Ciencias de la Salud) normalmente es el requisito básico e imprescindible para poder entrar en el proceso de selección" (E_Farmacia-2)․․

Solo hay una excepción en esta forma de proceder que afecta al inglés, al que en los sectores más competitivos como el de farmacia, se le da mucha importancia y, a pesar de disponer de titulación, se comprueba el nivel de dominio de la lengua porque valoran mucho esta competencia en un candidato: "[...] y por supuesto el inglés comprobarlo. He tenido alguna experiencia de que han contratado a alguien solo por el hecho de haber sido profesor de inglés teniendo un CV específico muy justo frente a otros candidatos" (E_Farmacia_1); "Llamada telefónica para verificar el conocimiento de idiomas" (E_Ingeniería_2).

Los empleadores entienden que, en su propósito de reclutar a los mejores candidatos, deben centrarse en evaluar el perfil personal o las competencias genéricas que son, sin duda, las que ellos más valoran: “[...] Depende del puesto de trabajo, pero en general las cualidades personales" (E_Ingeniería_2).

Básicamente, tal como te he comentado [hace referencia a las competencias personales o generales], en un informático valoramos como competencias generales las corporativas: cambio, sociabilidad, lideraje y análisis; y las funcionales. Dependerá del puesto de trabajo, pero básicamente serían capacidad de trabajo: ilusión, implicación, rigurosidad, efectividad, tenacidad, autonomía, seguridad, etc.; toma de decisiones: análisis, perfeccionismo, capacidad de improvisación, rapidez, etc.; flexibilidad: que sería adaptación al cambio, saber ajustarse a retos, tener iniciativa, ser un facilitador de las novedades, creatividad, etc.; capacidad de gestión, proyectos propios o de un equipo; capacidad de planificación y organización: visión a corto, medio y largo plazo, metodología de trabajo, etc.; sociabilidad, que serían las aptitudes para trabajar individualmente y en equipo. (E_Ingeniería_3).

Todas las citas textuales procedentes de las transcripciones textuales de los empleadores participantes en la investigación que se incluyen en este artículo se identifican de la manera siguiente: la letra E (empleador), el ámbito (Farmacia, Educación o Ingeniería) y el número de empleador de que se trata $(1,2,3)$, separados por guiones. 
En el ámbito educativo, los entrevistados manifiestan que las competencias de los maestros no están explicitadas desde la administración, con las implicaciones que de ello se derivan. Los empleadores de este sector perciben una necesidad de regulación de todos estos aspectos, y no parece que como responsables de reclutar a los maestros se sientan legitimados para decidir el perfil y los requisitos de los mismos, ni especialmente preocupados por su perfil personal, cuestiones ambas que contrastan con los otros sectores investigados. En este sentido, insisten en la falta de regulación concreta sobre las competencias del maestro.

No existe una normativa específica que establezca cuáles son las competencias profesionales del profesorado. En principio, la LEC, en el título VIII que dedica al profesorado, establece las funciones, y de aquí se podrían desprender algunas competencias. Se supone que estas se tendrían que desarrollar en el estatuto del profesorado, pero todavía no se ha hecho. Por otro lado, uno de los requisitos para acceder a la docencia en secundaria es el de poseer el máster de formación de profesorado. Si se consulta esta titulación en las webs de las universidades, se detallan las competencias profesionales; pero como es una titulación universitaria, la competencia legislativa recae en el departamento de universidades. (E_Educación_1).

Cuando especifican las competencias que se valoran en un maestro, se circunscriben a la formación que se ofrece en las universidades, o bien a aquellas que se evalúan desde la inspección, una vez que ya son maestros incorporados en la actividad de una escuela.

Como ya hemos dicho, cuando los empleadores hablan de las competencias que valoran en los candidatos, mayoritariamente, se circunscriben al ámbito de las competencias genéricas, que para ellos tienen que ver con habilidades, destrezas y capacidades que debe tener cualquier titulado antes de incorporarse al mercado laboral.

Dentro de las competencias genéricas, los resultados han sido categorizados siguiendo la propuesta del Proyecto Tuning (2007), en el que se identifican y definen tres tipos de competencias genéricas:

1. Competencias instrumentales: aquellas que tienen una función instrumental entre las que se incluyen habilidades cognoscitivas, metodológicas, tecnológicas y lingüísticas.

2. Competencias interpersonales: capacidades del individuo relativas a expresar los propios sentimientos, habilidades críticas y de autocrítica. Destrezas sociales relacionadas con las habilidades interpersonales, trabajo en equipo y compromiso social o ético.

3. Competencias sistémicas: destrezas y habilidades que suponen una combinación de la comprensión, la sensibilidad y el conocimiento que permiten al individuo ver cómo las partes de un todo se relacionan y se agrupan.

A la vista del análisis de la información recogida se observan competencias instrumentales que han valorado empleadores de todos los ámbitos y que son: la comunicación oral y escrita y la resolución de problemas. Es necesario matizar que, en general, la terminología utilizada por los empleadores alude más a la competencia comunicativa en sentido global, y no matizan tanto si dicha comunicación es escrita u oral: “[...] que a un profesional que se vaya a dedicar al ámbito asistencial [farmacia comunitaria o hospitalaria] donde lo que vamos a tener en cuenta es la orientación al paciente, las técnicas de comunicación [...]” (E_Farmacia_1).

En menor medida, aparecen competencias instrumentales tales como las habilidades del manejo de ordenador, que curiosamente aparece más en el ámbito educativo y no aparece 
en el de las ingenierías, quizás porque en este ámbito se les supone dicha competencia; la capacidad para organizar y planificar que figura en los ámbitos de ingeniería y farmacia y normalmente ligado, sobre todo, a la gestión del tiempo y no tanto a otro tipo de recursos; y, por último, la capacidad de análisis y síntesis que tampoco emerge en el ámbito educativo, y en los que ha emergido lo ha hecho en relación exclusivamente a la capacidad de reflexión y no a la de síntesis.

En cuanto al segundo grupo de competencias genéricas, las competencias interpersonales, la más valorada por los empleadores de los tres sectores, es la de trabajo en equipo, aunque es conveniente matizar el sentido que dan algunos de los empleadores a esta competencia, ya que además del aspecto colaborativo, le atribuyen una actitud positiva al candidato, hablando así de actitud positiva y de colaboración más que de trabajo en equipo propiamente dicho.

La capacidad crítica y autocrítica y el compromiso ético también se han identificado claramente por los empleadores, aunque con algunos matices: mientras la primera ha emergido en los 3 sectores, especialmente en el educativo, en cuanto al compromiso ético los empleadores del ámbito de farmacia sorprendentemente no han hecho referencia alguna. En cambio, los empleadores del ámbito educativo han hecho un especial énfasis en los aspectos éticos, relacionándolos con el hecho de tener una especial sensibilidad y compromiso hacia el entorno y la institución.

La competencia interpersonal, entendida como la capacidad para relacionarse con otros de manera apropiada, ha emergido en dos de los tres sectores: educación e ingeniería. A pesar de aparecer con menos insistencia, lo hace con mucha intensidad en las descripciones que la sitúan claramente en el ámbito emocional (competencias emocionales) y relacional.

Por último, las competencias sistémicas valoradas por los empleadores han sido las más subrayadas por los mismos. La capacidad de aprender y el liderazgo son las que aparecen con más frecuencia en los 3 sectores profesionales. Enfatizan, especialmente, la motivación de los candidatos por aprender, y no tanto los logros cognitivos.

La adaptación al cambio y la orientación al logro (a resultados, como ellos lo llaman) son las que aparecen en segundo lugar, aunque curiosamente no en todos los sectores analizados. La adaptación no emerge entre los empleadores del ámbito de farmacia, y sí en los otros, y la motivación de logro tampoco lo hace entre los de ingeniería.

Así mismo, la capacidad para generar nuevas ideas y la preocupación por la calidad no emergen con fuerza, ni tampoco lo hacen en todos los sectores, aunque desde un punto de vista cualitativo hay empleadores que le otorgan mucha importancia.

\subsubsection{Cómo evalúan los empleadores las competencias de los graduados (candidatos)}

Antes de adentrarnos en las estrategias y los instrumentos de evaluación de competencias que utilizan los empleadores, parece relevante puntualizar que la finalidad del proceso de evaluación que realizan los empleadores es seleccionar el mejor candidato según los requerimientos del cliente (empresa), según sus necesidades y su visión del puesto de trabajo a cubrir. Por lo tanto, en ningún momento se plantea la evaluación de competencias en este contexto como una estrategia para su desarrollo.

A la luz del discurso de los empleadores, podemos afirmar que hay una primera evaluación técnica o referida a competencias específicas que dejan en manos de las universidades, siendo su único cometido el comprobar que los candidatos poseen el título 
o tienen un determinado expediente o currículum, a partir de evidencias documentales como el título o el expediente académico. Si bien es cierto que todas ellas hacen referencia a datos de tipo académico, se puede distinguir el instrumento específico que utilizan los empleadores de cada ámbito: así, por ejemplo, mientras que el empleador del ámbito educativo utiliza el currículum (que contiene información amplia, tanto académica como profesional y personal); el del ámbito farmacéutico usa el currículum y la titulación (para acreditar competencias del programa requerido al final del proceso); y el profesional de ingeniería utiliza exclusivamente el expediente académico, y, en algún caso, de forma muy específica (teniendo en cuenta las calificaciones numéricas e incluso la calificación media numérica del expediente). En cualquier caso, los empleadores que consideran el currículum, tácitamente están considerando también el título que se ha obtenido. "En un profesional júnior o recién licenciado, $[\ldots]$ tanto mi cliente como yo damos por hecho que la titulación académica le acredita para desarrollar las funciones, o más bien, que cuenta con una base de conocimientos y herramientas para poder aprender las tareas de su nuevo puesto de trabajo [...]" (E_Farmacia_1); "Primero utilizo el expediente académico, con atención a la nota media y al índice alfa que es el porcentaje de asignaturas aprobadas sobre el total de matriculadas" (E_Ingeniería_4).

En el ámbito educativo, además, se hace referencia a toda aquella documentación escrita elaborada por el propio candidato que acredite o sirva de evidencia de su nivel de conocimiento de la materia y de las metodologías que utiliza, entre otras: "La documentación del centro y la documentación elaborada por el propio docente [graduado] para su intervención en el aula. [...], las producciones de los alumnos con los que ha trabajado" (E_Educación_3).

En este mismo orden de cosas, y siguiendo con el ámbito educativo, incluso manifiestan tener en cuenta el llamado currículum oculto: "La primera selección a través de Currículum; es importante poner atención en el CV oculto, las actividades de ocio, voluntariado, etc." (E_Educación_4).

Después de esta primera fase de evaluación de competencias específicas, a partir de evidencias documentales, algunos empleadores afirman realizar algunas valoraciones o comprobaciones suplementarias en relación a la preparación técnica en el ámbito profesional. Para ello, utilizan determinados instrumentos a modo de evidencias de la práctica profesional. Se trata de observaciones en el lugar de trabajo en, por ejemplo, los períodos de prácticas, o de pruebas técnicas suplementarias, algunas relacionando conocimientos técnicos y supuestos prácticos que hay que resolver: "[Se les pide] una respuesta escrita ante la presentación de un par de casos, explicando cuál sería su actuación. Se valora el criterio, el análisis, la empatía... [...]. Si es posible, invitar al candidato a asistir como persona de apoyo a una aula, y hacerle la observación" (E_Educación_4); "[...] y/o periodo de prácticas para evaluarle sobre el terreno, si es un recién Licenciado" (E_Farmacia_1); “A todos nuestros candidatos les pasamos pruebas técnicas para evaluar sus conocimientos técnicos [...]. Preguntas del estilo: qué harías si..." (E-Ingeniería_1).

A pesar de las coincidencias en aspectos tales como la valoración del título o del currículum en primer lugar, en el ámbito educativo, los instrumentos y el proceso evaluativo parecen tener unos matices que los hacen sensiblemente diferentes. A pesar de estar más reglamentada, y de contar con instrumentos más consensuados y sofisticados de evaluación, no parece que el resultado de la evaluación de los maestros noveles aporte mucha luz ni al propio evaluado ni a quien la realiza. Veamos la explicación de uno de los encuestados, 
inspector de educación en la Comunidad Autónoma de Cataluña:

Las evaluaciones docentes no están generalizadas como en otros países que existe la obligatoriedad de hacerlas cada cierto tiempo [...]. La evaluación de los docentes está organizada por la inspección de educación, que es quien tiene la competencia, por ahora. Se utiliza un instrumento diseñado por el Departament d'Ensenyament [Departamento de educación] que contiene una serie de indicadores agrupados en las diversas áreas que se evalúan. La evaluación es sólo positiva o negativa, pero no aporta más información. (E-Educación_1).

En cambio, sí que parece que la evaluación de los maestros en activo se realiza de manera sistemática y periódica, utilizando una multiplicidad de instrumentos para evidenciar el nivel de desarrollo competencial:

La Inspección cuando valora la actividad de un docente en el aula utiliza documentación del centro y la documentación elaborada por el propio docente para su intervención en el aula; producciones de los alumnos; observación directa de la actividad docente; entrevista personal; entrevista con el director del centro y entrevista con el coordinador de ciclo o Jefe de departamento. (E_Educación_3).

De igual manera, en el ámbito farmacéutico, uno de los empleadores también remarca la diferencia tanto del proceso de evaluación como del objeto de la misma cuando se trata de candidatos recién licenciados y candidatos ya con experiencia profesional. En el caso de los primeros, las competencias específicas se les suponen por disponer de una titulación universitaria, por tanto, estas no se evalúan y se centran en las competencias transversales y en las actitudes, o en lo que ellos denominan "perfil personal del candidato".

En cambio, cuando el candidato es "señor", sí que se hacen pruebas específicas de conocimientos, ya que se les exige una mayor calidad y profundidad en cuanto a los conocimientos técnicos. Concretamente, una de las encuestadas hace referencia a valorar las competencias del candidato en "registros farmacéuticos, desarrollo, transferencia, experiencia en ventas en determinado canal", entre otros. En estos casos, no se les preselecciona por la titulación, o no solamente por ella, sino por poseer un conocimiento profundo, a través de la experiencia profesional y de la especialización en un ámbito determinado. A partir de la comprobación de dichos conocimientos superiores, se prosigue con la evaluación del perfil personal de candidato.

Donde los empleadores focalizan fundamentalmente su discurso sobre las estrategias y los instrumentos de evaluación de competencias es en una herramienta de evaluación que consideran, todos ellos sin excepción, clave para la selección de un buen candidato y para la determinación de su perfil personal: la entrevista. "Lo primero, la entrevista personal en profundidad. [...] cuando selecciono a un recién licenciado en lo que más me fijo es en la persona [en la entrevista]. Lo que les hace obtener o no la plaza es su actitud, energía, proactividad, etc." (E_Farmacia-2); “[...] estas dos herramientas las completamos con una entrevista competencial, que es lo más importante (E_Ingeniería_3).

La finalidad principal de la entrevista, según los informantes, es conocer el perfil personal del candidato, sus reacciones ante determinadas situaciones, sus habilidades y sus características personales: "[...] y para evaluar más sus competencias personales les hacemos preguntas [en la entrevista] poniéndolos en situación para ver sus reacciones. 
Preguntas del estilo: qué harías si... o ¿crees que eres buen trabajador de equipo? Cuando has tenido que hacerlo, ¿trabajas bien bajo presión? [...] y este tipo de pregunta" (E_ Ingeniería_1).

Los adjetivos que utilizan los empleadores para referirse a las entrevistas ponen de manifiesto las características que le atribuyen: "personal" y entrevista "competencial". Es decir, la entrevista se erige como la estrategia principal para los empleadores en la construcción de un perfil personal del candidato en el que las características actitudinales y las competencias instrumentales son el objeto principal de su valoración.

Muchos de ellos, además, manifiestan que utilizan otros instrumentos, que se complementan con la entrevista, para la valoración del perfil personal del candidato, como por ejemplo los test comportamentales o psicotécnicos, que han sido mencionados por informantes de los ámbitos de farmacia e ingeniería, pero no por los de educación. Veámoslo: "Estas dos herramientas [se refiere a dos tipos de test psicotécnicos llamados PAPI _Cubicks_ y THOMAS] las completamos con una entrevista competencial." (E_Ingeniería_2); "Se utiliza el PROFILEXT, parecido al PAPI en cuanto a objetivos y evidentemente la entrevista competencial individual [...]” (E_Farmacia_3).

3.2. SEGUNDO BLOQUE DE RESULTADOS: EL PUNTO DE VISTA DE LOS EMPLEADORES EN RELACIÓN A LA ENSEÑANZA DE COMPETENCIAS EN LA UNIVERSIDAD

\subsubsection{Cómo creen los empleadores que se podría estimular el desarrollo de las competencias que ellos más valoran desde la universidad}

Los resultados obtenidos en respuesta a la tercera pregunta formulada nos han permitido agrupar las contribuciones de los empleadores en tres bloques que tienen que ver con los siguientes contenidos: 1. Medidas que deberían tomar las instituciones implicadas para mejorar el logro de competencias. 2. Competencias que se deberían enseñar en la universidad. 3. Medidas de tipo metodológico para favorecer el desarrollo de competencias en la universidad.

Antes de entrar a presentar las aportaciones correspondientes a cada uno de estos tres bloques, queremos señalar que, pese a que la pregunta que se formuló se refería a cómo creían los empleadores que se debería estimular en la universidad el desarrollo de las competencias que consideraran más relevantes, como veremos, sus respuestas se refieren a este "cómo" con propuestas sobre los cambios que deberían aplicar las instituciones implicadas, y, además, al tipo de competencias se deberían favorecer en la universidad. Estas aportaciones nos van a permitir establecer, más adelante, una comparación entre las competencias más valoradas desde los tres sectores profesionales y las que ellos mismos atribuyen a la universidad.

En relación con el primer bloque, Medidas que deberían tomar las instituciones implicadas para "mejorar el logro de competencias, desde Educación Primaria" (E_ Educación_1), un mismo empleador alude a la responsabilidad que debería asumir la Administración educativa en relación con la universidad con estas cuatro propuestas:

- La creación de un estatuto docente que defina y delimite cuáles tienen que ser las competencias comunes para todas las universidades.

- $\quad$ La realización de una evaluación sistemática y periódica en las universidades basada en las 
competencias del estatuto docente previamente consensuado.

- La realización de oposiciones con una exigencia mayor de la actual y diseñadas de acuerdo con las competencias profesionales.

- $\quad$ El aumento del tiempo dedicado al prácticum en los centros docentes.

En estas sugerencias, que son específicas del ámbito profesional de Educación Primaria, destaca la necesidad de que la administración educativa elabore un estatuto docente que defina cuáles deben ser las competencias docentes que sirvan de referente común para todas las universidades, y de que exista una coherencia entre estas competencias comunes de la formación universitaria y las que orienten el diseño de las oposiciones a maestro/a de Educación Primaria.

En cuanto a "Medidas que se deberían tomar desde la universidad, también desde Educación Primaria" (E_Educación_2), se sugiere una “selección más estricta del alumnado para el acceso a la universidad", aunque no se especifica qué tipo de instrumentos se emplearían para esta selección ni tampoco en relación a qué tipo de materias. Sin embargo, en cuanto a la competencia lingüística, las universidades públicas catalanas incentivarán el nivel de exigencia para el alumnado aspirante al Grado de Maestro de Educación Infantil y para el Grado de Maestro en Educación Primaria para el curso 2015-2016.

Además, uno de los empleadores de farmacia (E_Farmacia_1) también propone "Unos planes de estudios adecuados a lo que la sociedad va a demandar a los profesionales y la reducción del número de alumnos por aula".

Por último, por lo que respecta a las medidas que se deberían tomar entre la universidad y las empresas, en esta primera parte, dos empleadores del ámbito farmacéutico piden que la universidad se acerque a las demandas del mundo laboral y de las empresas, con propuestas como "La incentivación de los intercambios de conocimiento entre universidad y empresa y la contratación de profesorado con experiencia en las empresas y en la industria, además de la impartición de conferencias por parte de miembros de empresas" (E_Farmacia_1); "Un mejor conocimiento entre la realidad cotidiana en las empresas y las necesidades del mercado laboral y lo que se imparte en la universidad; charlas específicas de profesionales de la industria y la elaboración de los planes de estudios entre la universidad y las empresas" (E_Farmacia_2).

Entre estas medidas, cabe explicar que algunas de ellas ya son, en parte, un hecho. Por ejemplo, las plantillas de profesorado universitario empiezan a estar formadas por profesorado con experiencia en empresas a través de la figura de profesorado asociado, o la promoción en la universidad de más charlas específicas de profesionales del mundo de la empresa.

En cuanto al segundo bloque mencionado, las competencias que se deberían enseñar en la universidad, los empleadores consultados proponen competencias que hemos agrupado, siguiendo las orientaciones de Tuning (2007), en las mismas tipologías utilizadas para clasificar las competencias que los empleadores valoran más en un graduado:

- Competencias instrumentales, como las "Habilidades discursivas y argumentación en debates" (E_Educación_4; E_Ingeniería_2); "Comunicación" (E_Farmacia_3); "Exigencia personal y rigor en la resolución de las tareas" (E_Educación_4); "Resolución de problemas" (E_Educación_3); "Presentación pública de proyectos" (E_Ingeniería_2; E_Farmacia_2); "Organización y eficiencia y Gestión del tiempo" (E_Farmacia_3).

- Competencias interpersonales, como "el Aprendizaje cooperativo" (E_Educación_3); "Trabajos y proyectos en equipo" (E_Ingeniería_4; E_Ingeniería_2); "Trabajo en equipo exigente" (E_Farmacia_2). 
- Competencias sistémicas, como "la Autogestión de emociones y Autorregulación" (E_Educación_4); "Autoconocimiento para compensar déficits" (E_Educación_4); "Automotivación; Superación personal y Trabajo bajo presión" (E_Ingeniería_1); "Inteligencia emocional" (E_Ingeniería_3); "Liderazgo; Participación e implicación; Resiliencia" (E_Educación_4).

En cuanto al tercer bloque de esta tercera pregunta: Medidas de tipo metodológico para favorecer el desarrollo de competencias en la universidad, se identifican aportaciones referidas a una orientación metodológica de tipo general, como el "Método científico" (E_ Educación_4); "Definición y fomento de las competencias y Aumento de la capacidad para saber programar" (E_Ingeniería_1); "Diseño de actividades competenciales; Participación del alumnado en actividades y Aprendizaje basado en resolución de problemas y proyectos" (E_Educación_3).

Se sugieren, también, cierto tipo de actividades como "la Participación en debates" (E_Educación_4; E_Ingeniería_2); "Estudio de casos" (E_Educación_3; E_Ingeniería_3); "Discusión y debate sobre casos reales de empresas" (E_Ingeniería_3); "Presentaciones orales en público" (E_Farmacia_2); "Entrega de informes escritos" (E_Ingeniería_4); "Trabajos y proyectos realizados en equipo" (E_Ingeniería_4); "Role-playing" (E_ Farmacia_3; E_Ingeniería_3); "Dinámicas de grupo" (E_Ingeniería_3).

Destacamos que, desde Educación Primaria, se menciona el método científico y el diseño de actividades de carácter competencial. Desde Ingeniería se menciona la necesidad de intensificar las metodologías basadas en la práctica y que los graduados en informática sean competentes en programación. En cuanto a las concreciones en tipos de actividades, destacan las actividades prácticas y de aplicación del conocimiento sugeridas en mayor número por ingeniería y por farmacia.

\subsubsection{Cómo creen los empleadores que se deberían evaluar los aprendizajes y las} competencias de las y los estudiantes

El análisis de las respuestas a esta cuarta pregunta nos ha permitido agruparlas en los siguientes tres bloques: ¿Qué se debería evaluar desde la universidad?, ¿Cómo se debería evaluar en la universidad? y ¿Qué tipo de instrumentos se deberían usar en la universidad para evaluar?

Empezando por la pregunta: ¿qué se debería evaluar desde la universidad?, los empleadores han respondido, en primer lugar, con propuestas que coinciden en que se debe dar más importancia al conocimiento práctico, competencial y profesional cuando hablan de "Prácticum selectivo de acuerdo con los centros escolares, con valor de evaluación final del 40\%" (E_Educación_2); "Prácticas variadas en los centros" (E_Educación_3); "Aspectos teóricos y prácticos" (E_Ingeniería_2); "Proyecto de carrera" (E_Educación_3); "Conocimiento de las competencias profesionales" (E_Educación_1); "Conocimiento de las novedades en el mercado laboral” (E_Ingeniería_1). Y, en segundo lugar, con aportaciones de carácter competencial, en la misma línea de las competencias propuestas anteriormente en el apartado de Resultados, Las competencias valoradas por los empleadores en los graduados. Es decir, Instrumentales: "Comunicación” (E_Ingeniería_3); “Aplicación de conocimientos; Análisis" (E_Ingeniería_3); Interpersonales: "Negociación" (E_ Ingeniería_3); "Participación en equipo" (E_Ingeniería_3); “Implicación en la universidad, 
en la sociedad y en grupos de trabajo" (E_Farmacia_2). Sistémicas: "Emprendeduría" (E_Farmacia_2); "Pro-actividad e iniciativa" (E_Farmacia_2); "Autoconocimiento" (E_ Farmacia_2); "Liderazgo" (E_Ingeniería_3); "Rol en el grupo" (E_Ingeniería_3).

En cuanto a la pregunta ¿Cómo se debería evaluar en la universidad?, con bastante participación por parte de los tres ámbitos profesionales, todos ellos acentúan de nuevo el valor de la práctica y de las actividades desarrolladas en contextos reales. Los empleadores presentan dos tipos de contribuciones:

El primer tipo se relaciona con criterios y estrategias de evaluación, que tienen que ver con "valorar no sólo lo que saben, sino lo que saben hacer" (E_Educación_3); "el Rigor en la evaluación y Conocimiento por parte del alumnado de los criterios de evaluación" (E_Educación_4); "la superación de todos los aprendizajes y la consideración de que las competencias no están superadas si obtienen nota inferior al 60\%" (E_Educación_2); “Como en el mundo empresarial, descubriendo puntos fuertes que se deben maximizar y saber para qué son útiles y de puntos débiles, para reforzarlos" (E_Farmacia_3); "la evaluación de competencias profesionales por parte de la dirección de los centros” (E_Educación_1).

El segundo tipo se vincula con aspectos a evaluar. A partir de situaciones reales como el "Prácticum en los centros selectivos, con evaluación estricta y buen seguimiento y las prácticas como el mejor instrumento evaluativo" (E_Educación_3); "La práctica, de acuerdo con lo que pide la empresa" (E_Ingeniería_1); "la evaluación de competencias profesionales docentes con más práctica directa" (E_Educación_1); "la evaluación continua en situaciones reales sobre el nivel de conocimientos, aptitudes y habilidades" (E_Farmacia_1).

En relación con la tercera parte de las respuestas a esta cuarta pregunta: ¿Qué tipo de instrumentos se deberían usar en la universidad para evaluar?, los tres grupos de empleadores sugieren algunos instrumentos que ya son de uso habitual en ámbitos académicos, como "cuestionarios y desarrollo de respuestas de forma oral y escrita" (E_Educación_4); "Mapas conceptuales" (E_Educación_4); “Aspectos teóricos mediante examen, aspectos prácticos de forma continuada y por resultados" (E_Ingeniería_2); "Rúbricas de evaluación sencillas, para evaluar sólo lo más significativo" (E_Ingeniería_1).

A excepción de las aportaciones de farmacia, referidas a la aplicación de "Test comportamentales y creación de servicios de coaching de competencias, complementario del servicio de orientación profesional, para trabajar puntos de apoyo y vigilancia" (E_ Farmacia_2); "Creación de centros de simulación para exponer al alumno a experiencias de evaluación" (E_Farmacia_1).

\subsubsection{Otras consideraciones de los empleadores}

En relación con la quinta y última pregunta abierta, los resultados se concentran aportaciones correspondientes a propuestas de cambio en la enseñanza superior.

En primer lugar, identificamos contribuciones de Educación Primaria referidas al Diseño de los estudios universitarios, para los que se identifican tres cuestiones: "La ampliación de créditos y la realización de un proyecto final" (E_Educación_4), y "La modificación de los estudios con un currículum basado en las competencias expuestas (véase las aportaciones a la pregunta 1)" (E_Educación_3).

En segundo lugar, contribuciones para la evaluación post gradual, en donde se agrupan propuestas relativas a una evaluación previa al ejercicio profesional. Por parte de Educación 
Primaria se propone un sistema de formación de especialidad que evalúe, en la práctica, las competencias profesionales antes de ejercer la profesión, a través de la "realización de una interinidad que permita obtener las competencias profesionales con la práctica" (E_Educación_1). O bien a lo largo del ejercicio profesional, a través de la "evaluación por sexenios y aplicación de resultados para la promoción docente" (E_Educación_1), medida esta que, sin duda, estimularía el mantenimiento y el desarrollo competenciales.

Por último, en la relación universidad-empresa, se identifican aportaciones que inciden, de nuevo, y de manera especial, en la necesidad de adaptación de la universidad a las demandas de la profesionalización y a una formación del alumnado que tenga en cuenta tanto los conocimientos como las aptitudes prácticas y conductuales. En este sentido, los empleadores hablan de "no disrupción entre el mundo académico y el laboral" (E_ Farmacia_3); "Mayor proximidad a la sociedad, a la economía y a la realidad de las empresas, fomentando más las aptitudes y no sólo el conocimiento académico" (E_Ingeniería_3); "Aprendizaje desde la universidad de lo que es importante en el mundo laboral desde un punto de vista pragmático" (E_Farmacia_3); "Demostración de habilidades conductuales y de potencial como profesionales de futuro" (E_Ingeniería_3); o "Que el estudiante conozca lo que le pedirán y le valorarán en el futuro profesional" (E_Farmacia_3).

En cualquier caso, también se produce un reconocimiento explícito de la evolución de la universidad en esa dirección, cuando manifiestan creer con firmeza "que la Universidad ya se viene moviendo en la buena dirección para acercarse más a la sociedad, a la economía y a la realidad de las empresas, aportando mayores conocimientos y aptitudes a los alumnos más allá del conocimiento académico, que es fundamental y nunca debe dejar de ser el foco principal" (E_Farmacia_2).

\section{DISCUSIÓN Y CONCLUSIONES}

La información proporcionada por los empleadores confirma la ambigüedad e imprecisión con que tiende a usarse el término competencia (Lévy-Leboyer, 2003) y la falta de consenso existente en torno al mismo (Tardif, 2008). Para la Comisión Europea (2004, p. 5), el concepto de competencia alude a "una combinación de destrezas, conocimientos, aptitudes y actitudes, y a la inclusión de la disposición para aprender, además de saber cómo". Esta concepción, asumida por gran parte de la comunidad académica, pone el énfasis en la articulación de estos elementos y en "un saber actuar complejo que se apoya sobre la movilización y la utilización eficaz de una variedad de recursos" (Tardif, 2008, p. 3). Sin embargo, los empleadores de nuestro estudio le atribuyen un significado más ligado a las capacidades, habilidades y actitudes de los candidatos.

Por otra parte, lo que el contexto universitario considera competencias específicas del ámbito profesional, para los empleadores es la preparación conceptual y técnica que se presupone ha adquirido el candidato durante la formación universitaria, pero no las entienden como competencias.

En un profesional júnior o recién licenciado, como indican bastantes respuestas al cuestionario, tanto mi cliente como yo, damos por hecho que la titulación académica le acredita para desarrollar las funciones, o más bien, que cuenta con una base de conocimientos y herramientas para poder aprender las tareas de su nuevo puesto de trabajo según vaya adquiriendo experiencia en el mismo. (E_Farmacia_1). 
Los empleadores valoran, principalmente, las competencias genéricas, concretamente las competencias interpersonales (entre las más valoradas, el trabajo en equipo) y sistémicas (el liderazgo) y, en menor medida, las instrumentales. En este sentido, se aprecia cierta similitud con los resultados de la investigación realizada por Freire et al. (2011) que confirma a las competencias sistémicas como las más valoradas en el mercado laboral (responsabilidad en el trabajo, capacidad de aprender, motivación por el trabajo, preocupación por la calidad, etc.) y, entre las interpersonales, destaca también el trabajo en equipo. Competencia esta última igualmente subrayada en las investigaciones realizadas por Palmer et al. (2009), Nelcy et al. (2012) y Jackson (2012).

Estos datos evidencian cierta escisión entre el mundo académico y el ámbito empresarial, pues mientras este, tal y como se constata en el estudio, resalta el valor de las competencias generales, desde la formación universitaria, quizá porque tradicionalmente ha sido así, se incide más en las competencias específicas. Es precisamente este hecho el que, en opinión de Corominas et al. (2006), ayuda a entender la reticencia del profesorado a incorporar las competencias generales en el currículum académico. También Palmer et al. (2009, p. 438) se hacen eco de esta escisión cuando en las conclusiones de su investigación afirman: "Es interesante destacar que en la selección realizada por los empleadores no aparecen las competencias que tradicionalmente han formado parte del objetivo de la formación universitaria". Es posible que esto se deba, como se ha comentado antes, a que los empleadores asumen que los graduados ya han desarrollado las competencias específicas durante su formación universitaria.

Esta consideración se ve alterada en casos muy concretos, como el de lengua extranjera, en el que los empleadores tienden a incluir algún tipo de prueba para constatar las competencias del alumnado vinculadas a esta cuestión. Esta excepcionalidad refleja la importancia conferida por los empleadores al dominio de una o varias lenguas extranjeras y la desconfianza hacia la formación proporcionada por la institución universitaria en este punto. Como ha puesto de manifiesto la investigación llevada a cabo por Nelcy y otros (2012), hablar y escribir en un idioma extranjero es una de las pocas competencias en las que el nivel de competencia adquirido está por debajo de las expectativas y demandas de los empleadores.

En general, los empleadores tienden a dar mucha importancia a la dimensión práctica de la competencia, al "saber hacer", pero aluden poco a la vertiente más teórica o conceptual. Es importante destacar que en ningún caso mencionan la relación teoría-práctica, aunque en la universidad esta conexión se considera clave para garantizar un correcto desarrollo competencial. Situaciones como estas son las que llevan a algunos autores a demandar un mejor encaje entre la formación universitaria y el mercado laboral (Alonso et al., 2009; Jackson, 2012; Torres-Coronas y Vidal-Blasco, 2015), tal como aparece en este estudio.

Respecto a la evaluación, los empleadores le adjudican una finalidad básicamente acreditativa, de control, pues la consideran un proceso de selección que no solamente acredita el desarrollo competencial de una persona, sino que permite seleccionar el mejor candidato. En este sentido, podría decirse que la evaluación realizada por los empleadores está cercana, en cuanto a su finalidad, al discurso dominante que ha regido la actividad evaluadora tradicionalmente en la universidad, una evaluación orientada a resultados, selectiva y de control (Ibarra y Rodríguez, 2010). Sin embargo, en la universidad se aprecia una incipiente evolución hacia procesos de evaluación más formativos, que conviven con las viejas tendencias, dando lugar a ciertas disonancias, aún no resueltas, que Margalef (2014, 
p. 44) recoge en su investigación cuando afirma: “detectamos claramente las paradojas que surgen entre dos concepciones de evaluación: una formativa, centrada en la calidad de la experiencia vivida y en el proceso de aprendizaje, y otra, acreditativa, con fuerte peso en la especificación de resultados".

Por otra parte, la evaluación de los empleadores discrepa, en cuanto a sus condicionantes, de la evaluación realizada en la universidad, pues, mientras la evaluación en el ámbito empresarial está condicionada por las necesidades del contexto, la evaluación en la universidad ha estado centrada en el conocimiento, no siempre cercano a las necesidades del contexto laboral, aunque el Proceso de Convergencia está sirviendo de detonante para estrechar lazos entre el ámbito académico y laboral.

$\mathrm{Si}$ nos centramos en los instrumentos y estrategias de evaluación que usan los empleadores, se constata el protagonismo absoluto de la entrevista. Algunos aluden a un tipo de entrevista concreto que denominan "personal y/o competencial" y que les ayuda a determinar el perfil personal del candidato, entre otros aspectos. En ese trabajo se subraya el valor de la entrevista como instrumento para recabar información y validar los datos obtenidos a través del currículum o la realización de pruebas psicotécnicas o test de personalidad, entre otros. Los empleadores, de manera unánime, proponen la adopción de métodos y enfoques de evaluación más centrados en los aspectos prácticos y rasgos personales de los aspirantes (honestidad, empatía, sinceridad, entre otros), coincidiendo así con lo subrayado en los trabajos de Ahmad et al. (2010) y Bhanugopan y Fish (2009). Esto implica la adopción de nuevos enfoques, distintos al tradicional, tal y como han subrayado Gulikers et al. (2010) y Villarroel y Bruna (2014).

En opinión de los empleadores, el desarrollo de competencias en la universidad requiere de un trabajo coordinado entre administración, universidad y empresas, como también subraya Jackson (2012). Esta coordinación conlleva cambios estructurales que afectan a la relación que mantiene la administración educativa con las universidades y a la de estas con las empresas. Los cambios estructurales que proponen los empleadores responden a decisiones que deben adoptarse en diferentes momentos: antes de realizar los estudios universitarios, durante la realización de los estudios y otras que implican mejoras en la formación continua y en la evaluación pos-gradual.

En el marco de las decisiones previas a la realización de los estudios superiores, los empleadores plantean, por ejemplo, la creación de un estatuto docente que sea asumido por todas las universidades, incrementar el tiempo dedicado al prácticum, reducir el número de alumnos por aula, endurecer los criterios de acceso y los procesos de selección, revalorizar la importancia de la formación práctica y la aplicación del conocimiento en los planes de estudios y en los programas de formación docente y que, estos últimos, se elaboren de acuerdo con las necesidades reales del desempeño profesional.

Algunas de las propuestas sugeridas por los empleadores para llevarlas a cabo durante los estudios ya se realizan actualmente en las universidades, aunque de forma desigual según las titulaciones. Nos estamos refiriendo a sugerencias como la elaboración de un proyecto fin de carrera, la promoción de intercambios de conocimiento entre universidad y empresa, la contratación de profesorado con experiencia en el ámbito laboral, o la participación puntual, mediante conferencias, de personal de empresas. Otras aportaciones, más atrevidas, apuntan a una participación activa y directa en la elaboración de los temarios entre universidad y empresa. Algunas de estas propuestas han sido sugeridas por Bhanugopan y Fish (2009) y Parris y Saville (2011). Estos últimos, por ejemplo, subrayan la 
conveniencia de ampliar las prácticas en empresas, estrechar los vínculos con las empresas y reforzar el tratamiento de los aspectos prácticos de la formación en los planes de estudios. En esta dirección se pronuncian los participantes en el estudio llevado a cabo por la Unidad de Calidad de la Universidad de Alicante cuando señalan

[...] la necesidad de incrementar las prácticas en empresa y a su vez también en la participación de las empresas en seminarios y actividades que permitan a los empleadores aportar la visión de la empresa a los estudiantes. Entre otras vías proponen la participación de profesionales en seminarios y un impulso de la figura del profesor asociado. (2010, p. 72).

Con respecto a las sugerencias formuladas por los empleadores para mejorar la formación y evaluación pos-gradual, destacamos, en el caso del Grado en Educación Primaria, la realización de evaluaciones sistemáticas, cada seis años, basadas en las competencias recogidas en el estatuto docente, incrementar el grado de exigencia en la revisión y realización de oposiciones de acuerdo con el marco competencial, la existencia de un período de interinidad para consolidar el logro de competencias, la creación de centros de simulación para ejercitarse en la resolución de casos, un servicio de orientación profesional sobre competencias, o la evaluación de competencias por parte de la dirección de las empresas.

En relación con las competencias que se deberían desarrollar en la universidad, los empleadores se refieren a los tres tipos de competencias genéricas ya subrayadas (instrumentales, interpersonales y sistémicas), con importantes coincidencias. Entre ellas destacamos: las habilidades discursivas orales y escritas, la resolución de problemas, la capacidad organizativa y la eficiencia, el trabajo en equipo, la tolerancia a la presión o el liderazgo. Como han recogido Jackson (2012) y Freire et al. (2011) en sus trabajos, se trata de competencias muy valoradas en el sector laboral, pero no siempre suficientemente cultivadas desde la formación universitaria. Resulta llamativo que no haya alusión a las competencias específicas como parte también importante de la formación universitaria. Quizá este hecho guarde relación con la mercantilización del conocimiento y su devaluación, por tratarse de un bien efímero, como señala Bauman (2008), aunque existe la posibilidad de que este olvido sea consecuencia de la creencia arraigada que lleva a sobrentender que esa formación es labor de la institución universitaria.

En opinión de los empleadores de nuestro estudio, las metodologías que más favorecen el desarrollo de competencias en la universidad son aquellas que se centran en la práctica, con actividades que acercan al alumnado a casos reales, de manera simulada o favoreciendo el contacto directo con la práctica, y que fomentan el trabajo en equipo. Se reconoce así el impacto positivo que tendría incorporar a la formación universitaria experiencias de trabajo bien planificadas y estructuradas, consideración esta también subrayada por Taylor y Hooley (2014) y la Unidad Técnica de Calidad de la Universidad de Alicante (2010).

En coherencia con esta postura, los empleadores del estudio proponen que la evaluación de competencias en la universidad se centre en la propia práctica, en la realización de proyectos, además de valorar la iniciativa, implicación del alumnado, liderazgo y rol en el grupo, y la actualización de conocimientos. Para ello proponen combinar los instrumentos de evaluación tradicionales (exámenes, cuestionarios, mapas conceptuales, etc.) con otros procedimientos e instrumentos como la observación del alumnado en situaciones prácticas (reales o simuladas) y las rúbricas. En este sentido, se muestran a favor de una diversificación 
de los procedimientos e instrumentos de evaluación, contraviniendo, en parte, la posición de los empleadores en otros estudios nacionales y europeos que consideran que los exámenes no juegan un papel importante en la adquisición y desarrollo de competencias (Alcañiz, Claveria y Riera, 2013; Schomburg y Teichler, 2007).

\section{REFERENCIAS BIBLIOGRÁFICAS}

Ahmad, S., Ishak, N. M., Ismail, K., \& Selamat, J. (2010). Generic competency profile among students in institute of higher learning: A case of Universiti Kebangsaan Malaysia. College Student Journal, 44(3), 811-820.

Alcañiz, M., Claveria, O., \& Riera, C. (2013). Competencias en educación superior desde tres perspectivas diferentes: estudiantes, empleadores y académicos. Revista Iberoamericana de Educación, 66(2), 1-19.

Alonso, L.E., Fernández, C., \& Nyssen, J.M. (2009). El debate sobre las competencias. Una investigación cualitativa en torno a la educación superior en España. Madrid: ANECA.

Baartman, L., Prins, F., Kirschner, P., \& Van Der Vleuten, C. (2007). Determining the quality of competence assessment programs: A self-evaluation procedure. Studies in Educational Evaluation, 33(3-4), 258-281.

Bauman, Z. (2008). Los retos de la educación en la modernidad líquida. Barcelona: Gedisa.

Bhanugopan, R., \& Fish, A. (2009). Achieving graduate employability through consensus in the south pacific island nation. Education \& Training, 51(2), 108-123.

Cano, M.E. (2008). La evaluación de competencias en la educación superior. Profesorado. Revista de currículum y formación del profesorado, 12(3), 1-16.

Colás, P., \& Buendía, L. (1998). Investigación educativa. Sevilla: Ediciones Alfar.

Comisión Europea. (2004). Competencias clave para un aprendizaje a lo largo de la vida. Un marco de referencia europeo. Madrid: Dirección General de Educación y Cultura. Recuperado de http://www.educastur.princast.es/info/calidad/indicadores/doc/comision_europea.pdf

Corominas, E., Tesouro, M., Capell, D., Teixidó, J., Pèlach, J., \& Cortada, R. (2006). Percepciones del profesorado ante la incorporación de las competencias genéricas en la formación universitaria. Revista de Educación, 341, 301-336.

Freire, M.J., Teijeiro, M., \& Pais, C. (2011). Políticas educativas y empleabilidad: ¿cuáles son las competencias más influyentes? Archivos Analíticos de Políticas Educativas, 19(28), 1-24. Recuperado de http://epaa.asu.edu/ojs/article/view/911

García-San Pedro, M.J., \& Gairin, J. (2011). Los mapas de competencias: Una herramienta para mejorar la calidad de la formación universitaria. Revista Iberoamericana sobre Calidad, Eficacia y Educación, 9(1), 84-102.

Gulikers, J., Baartman, L., \& Biemans, H. (2010). Facilitating evaluations of innovative, competencebased assessments: Creating understanding and involving multiple stakeholders. Evaluation and Program Planning, 33(2), 120-127.

Ibarra, M.S., \& Rodríguez, G. (2010). Aproximación al discurso dominante sobre la evaluación del aprendizaje en la universidad. Revista de Educación, 351, 385-407.

Jackson, D. (2012). Business undergraduates' perceptions of their capabilities in employability skills: Implications for industry and higher education. Industry and Higher Education, 26(5), 345-356.

Jenkins, A. (2000). It's a long hard road! In S. Fallows \& C. Steven (Eds.), Integrating key skills in higher education (pp. 183-200). London: Kogan Page.

Kellermann, P. (2007). Acquired Competences and Job Requirements. In U. Teichler (Ed.), Careers of university graduates: views and experiences in comparative perspectives (pp. 115-130). The Netherlands: Springer. 
Lévy-Leboyer, C. (2003). Gestión de las competencias: cómo analizarlas, cómo evaluarlas, cómo desarrollarlas: Barcelona: Gestión 2000.

Margalef, L. (2014). Evaluación formativa de los aprendizajes en el contexto universitario: resistencias y paradojas del profesorado. Educación XX1, 17(2), 35-55.

Mcmurray, I., Roberts, P., Robertson, I., \& Teoh, K. (2011). An action research project exploring the psychology curriculum and transitions to employment. Psychology Teaching Review, 17(1), 50-63.

Nelcy, M., Rodríguez, P., Hincapié, J., Aguadelo, A.M., \& Ramírez, R. (2012). Percepción de empleadores sobre las competencias de graduados del Programa de Enfermería de la Fundación Universitaria del Área Andina de Pereira (Colombia). Revista Cultura del Cuidado, 9(2), 22-38.

Palmer, A., Montaño, J.J., \& Palou, M. (2009). Las competencias genéricas en la educación superior. Estudios comparativo entre la opinión de empleadores y académicos. Psicothema, 21(3), 433438.

Parris, M., \& Saville, K. (2011). Piecing together the puzzle of graduate employment: Factors that shape the graduate work expectations of human resource management students. Industry and Higher Education, 25(1), 15-24.

Pozo, C., \& Bretones, B. (2015). Dificultades y retos en la implantación de los títulos de grado en las universidades españolas. Revista de Educación, 267, 147-172.

Proyecto Tuning. (2007). Tuning education structures in Europe. Informe final. Bilbao: Universidad de Deusto.

Rutt, L., Gray, C., Turner, R., Swain, J., Hulme, S., \& Pomero, R. (2013). A social constructivist approach to introducing skills for employment to foundation degree students. Research in PostCompulsory Education, 18(3), 280-296.

Schomburg, H., \& Teichler, U. (2007). Higher education and Graduate Employment in Europe. The Netherlands: Springer.

Tardif, J. (2008). Desarrollo de un programa por competencias: de la intención a la implementación. Profesorado. Revista de Currículum y Formación del Profesorado, 12(3), 1-16. Recuperado de http://www.ugr.es/ recfpro/rev123ART2.pdf

Taylor, A.R., \& Hooley, T. (2014). Evaluating the impact of career management skills module and internship programme within a university business school. British Journal of Guidance \& Counselling, 42(5), 487-499.

Torres-Coronas, T., \& Vidal-Blasco, M.A. (2015). Percepción de estudiantes y empleadores sobre el desarrollo de competencias digitales en Educación Superior. Revista de Educación, 367, 63-90.

Tran, T.T. (2013). Limitation on the development of skills in higher education in Vietnam. Higher Education: The International Journal of Higher Education and Educational Planning, 65(5), 631-644.

Unidad Técnica de Calidad de la Universidad de Alicante. (2010). Competencias y habilidades del alumnado egresado de la Universidad de Alicante. La visión de los empleadores. Alicante: Universidad de Alicante.

Velde, C. (2009). Employers' perceptions of graduate competencies and future trends in higher vocational education in china. Philadelphia: Routledge.

Villarroel, V., \& Bruna, D. (2014). Reflexiones en torno a las competencias genéricas en educación superior: Un desafío pendiente. Psicoperspectivas, 13(1), 23-34.

Watson, R. (2011). A rationale for the development of an extracurricular employability award at a British University. Research in Post-Compulsory Education, 16(3), 371-384.

Wickramasinghe, V., \& Perera, L. (2010). Graduates', university lecturers' and employers' perceptions towards employability skills. Education \& Training, 52(3), 226-244. 
